\title{
As Influências dos Valores Pessoais nas Atitudes e nas FASES DA Lealdade
}

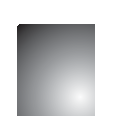

\author{
The Influence of Personal Values on Attitudes and Loyalty \\ Stages
}

Jorge Luiz Henrique

Gestor no Banco do Brasil - Porto Alegre - RS - Brasil. E-mail: jl.henrique@uol.com.br

Plínio Rafael Reis Monteiro

Professor dos cursos de Mestrado e Doutorado em Administração da Universidade FUMEC - Belo Horizonte - MG - Brasil. E-mail: p.monteiro@institutoanalysis.com.br

Celso Augusto de Matos

Professor do Programa de Pós-Graduação em Administração da Universidade do Vale do Rio dos Sinos - São Leopoldo - RS - Brasil. E-mail: celsoam@unisinos.br

\section{Resumo}

A lealdade é um tema bastante investigado no marketing, com pesquisas que avaliam como as diferenças entre consumidores (exemplo perfil demográfico) influenciam as fases da lealdade. Apesar disso, nota-se uma lacuna na investigação das relações entre valores pessoais, as fases da lealdade $e$ as atitudes do consumidor. Com o objetivo de testar o papel moderador dos valores pessoais na relação entre as atitudes $e$ as fases da lealdade de Oliver (1999), foi realizado um survey com 910 clientes bancários. Os resultados apontaram que as atitudes antecedem a lealdade, apesar de as diferenças entre os segmentos (Autopromocionais, Hedônicos e Autotranscendentes) não se mostrarem significativas. No entanto, na comparação de médias de lealdade dentre os segmentos, pode-se destacar que (i) os autopromocionais se diferenciam dos autotranscendentes, nas fases cognitiva e afetiva, e (ii) os autopromocionais se diferenciam dos hedônicos na fase ação. Por fim, são discutidas as implicações teóricas, as gerenciais e as sugestões para pesquisas futuras.

Palavras-chave: Atittudes. Personal Values. Loyalty.

\section{Abstract}

Although loyalty has been extensively investigated in the marketing discipline, there is a lack of attention to the relationship between personal values, loyalty and attitudes. Based on that, the objective of this study was to test the moderating role of personal values in the relationship between attitudes and loyalty stages from Oliver (1999). Data from a survey with 910 bank customers support the hypothesis that attitudes predicts loyalty, despite the structural model does not reveal statistically significant differences among customer clusters (self-promotional and selftranscending Hedonic). However, when comparing Loyalty means among segments, it can be noted that (i) the self-promotional and self-transcendent clusters were different at the cognitive and affective stages, and (ii) the self-promotional differed from the hedonic in the action stage. Finally we discuss the theoretical and managerial implications and provide suggestions for future research in the area.

Key words: Atitudes. Valores Pessoais. Lealdade.

Palavras-chave: Atittudes. Personal Values. Loyalty. 


\section{INTRODUÇÃo}

As estratégias de marketing das organizações e as análises sobre o comportamento do consumidor mudaram ao longo dos anos, evoluindo da busca da satisfação dos clientes e do atendimento de seus desejos na relação transacional para a formação de relacionamentos de longo prazo. Essa mudança de postura do marketing (BAGOZZI, 1974), o aumento da fragmentação dos mercados (YIM; KANNAN, 1999) $e$ a evolução do marketing de relacionamento favoreceram a busca pela lealdade do consumidor. Assim, os estudos realizados sobre a lealdade - considerada uma repetição de compra induzida, não aleatória, de uma marca específica, a partir de um conjunto de alternativas ao longo do tempo por um consumidor (JACOBY; KYNER 1973) - foram intensificados. No entanto, a lealdade é ainda um constructo que não apresenta uma universalidade quanto à sua definição conceitual (RUNDLE-THIELE; MACKAY, 2001), nem sobre como esse constructo deve ser medido (BENNETT; RUNDLE-THIELE, 2002). Chaudhuri e Holbrook (2001) constataram que a maioria dos estudos envolvendo conceitos de lealdade enfatizava a dimensão comportamental, negligenciando os componentes atitudinais e seus relacionamentos com outras variáveis, tanto do consumidor quanto do mercado. Assim, constata-se que há relevância em buscar novos antecedentes e moderadores da lealdade, como, por exemplo, atitudes e valores pessoais.

As atitudes podem ser utilizadas para prever futuros comportamentos (SHETH; MITTAL; NEWMAN, 2001). Logo, conhecer as atitudes do indivíduo implica conhecer um pouco mais sobre o consumidor e prever seu comportamento frente a uma situação específica. Na literatura, encontram-se alguns estudos que investigaram as atitudes como antecedentes do comportamento (ROHAN, 2000; KIM et al., 2002; MARANDI; LITTLE; SEKHON, 2006). As atitudes são, ainda, expressões de um determinado valor em relação a um objeto ou à ideia específica (LESSIG; COPLEY, 1974). Porém, as atitudes são diferentes dos valores. (GRUBE; MAYTON, II; BALL-ROKEACH, 1994)

Os valores pessoais são crenças únicas que transcendem objetos e situações, pois têm um papel importante nas representações das necessidades $e$ dos desejos individuais, de um lado, e por deman- das sociais, do outro (GRUBE; MAYTON, II; BALL-ROKEACH, 1994). Há algum tempo, os valores pessoais são tratados como um constructo importante na compreensão do comportamento do consumidor (BEATTY et al., 1985; LAGES; FERNANDES, 2005). Assim, considerando que a lealdade é composta por questões comportamentais (JONES; TAYLOR, 2007), constata-se que, como as atitudes, os valores pessoais podem prever a lealdade do consumidor.

A conexão entre esses três contructos foi investigada por Marandi, Little e Sekhon (2006) que confirmaram o impacto dos valores pessoais nas atitudes e a influência dessas na lealdade dos clientes de um provedor de serviços. No entanto, não há estudos empíricos que tratam os valores pessoais como moderadores na relação entre atitudes e as fases da lealdade, propostas por Oliver (1997, 1999). A relevância da investigação dessa moderação está no fato de os valores pessoais serem ligados à emoção, às situações motivacionais $e$ à avaliação de ações e eventos (SCHWARTZ, 1992, 2006), podendo, dessa forma, os valores afetarem a força da relação entre atitudes (constructo independente) $e$ as fases da lealdade (constructo dependente). Por exemplo, um indivíduo que possua o valor liberdade, como característica, pode agir de forma positiva em relação a uma marca e, consequentemente, permanecer comprando essa marca (lealdade conativa).

Assim, o objetivo deste estudo é testar e comparar diferentes valores pessoais como moderadores na relação entre atitudes e fases da lealdade de Oliver (1999). Dessa forma, este estudo busca ampliar o conhecimento sobre antecedentes e moderadores das fases da lealdade.

O trabalho está dividido em cinco seções: fundamentação teórica, modelo teórico, metodologia, resultados e discussões/considerações finais.

\section{Fundamentação Teórica}

Neste tópico são debatidos e discutidos os temas centrais que permeiam a concepção $e$ a aplicação do estudo. Inicia-se pela retomada do tema de lealdade e atitudes no contexto de consumo, finalizando pela reflexão dos valores pessoais como elemento moderador central para compreender as reações emocionais, cognitivas e conativas em diferentes contextos mercadológicos. 


\subsection{Lealdade}

A lealdade é definida como um profundo compromisso de recompra de um produto ou serviço a despeito de influências situacionais ou esforços de marketing que tenham potencial de causar um comportamento de mudança. (OLIVER, 1997, 1999)

A busca pela lealdade do consumidor intensificou-se a partir da mudança de postura do marketing, que passou do estudo das relações de troca (BAGOZZI, 1974) para o estudo de relacionamentos duradouros (DWYER; SCHURR; OH, 1987). Esse foco relacional motivou um incremento nas investigações tanto sobre antecedentes e consequentes da lealdade, dentre eles satisfação (GARBARINO; JOHNSON, 1999), confiança e comprometimento (MORGAN; HUNT, 1994) e valor (FORNELL et al., 1996), quanto sobre as abordagens conceituais do constructo, comportamental (SEIDERS et al., 2005) ou atitudinal (JACOBY; CHESNUT, 1978), além de estudos sobre o modelo proposto por Oliver (1997, 1999), das fases da lealdade - cognitiva, afetiva, conativa e ação. (HARRIS; GOODE, 2004)

Oliver $(1997,1999)$ afirma que cada fase do modelo proposto está baseada em aspectos comportamentais e/ou atitudinais. A fase cognitiva está vinculada às informações, tais como preços, qualidade e atributos de produtos. Nesta fase, o consumidor prefere uma marca em relação às demais do mercado. Assim, a lealdade, baseada em crenças e conhecimento, assume um caráter superficial. A fase afetiva está vinculada ao gosto, ao sentimento e à satisfação do cliente. Nesta fase, o compromisso tem uma atitude positiva (afeto) em relação à marca. A lealdade ainda é superficial e dependente da força afetiva da marca. A fase conativa está vinculada à intenção comportamental e ao comprometimento do consumidor. Nesta fase, o consumidor é influenciado pelas sucessivas experiências positivas de compra e de afeto em relação à marca. A lealdade é mais forte, sustentada pelo comprometimento e pela intenção de comprar. A fase ação está vinculada à predisposição de agir e à superação de obstáculos que impeçam a compra do produto/serviço por parte do consumidor. O modelo de Oliver foi investigado por McMullan e Gilmore (2003); Harris e Goode (2004); Caruana (2004); Lu et al. (2005) e Evanschitzky e Wunderlich (2006).

\subsection{Atitudes}

As atitudes são organizações relativamente duradouras de crenças existenciais, avaliativas, prescritivas e causais de um objeto ou de uma situação. Essas atitudes têm propriedades motivacionais que predispõem os indivíduos a responder a um objeto ou situação e/ou às pessoas envolvidas nessas situações, independentemente de pressões ou de controles sociais. (GRUBE; MAYTON, II; BALL-ROKEACH, 1994)

As atitudes são suportadas pelos valores (ROKE$\mathrm{ACH}, 1981)$ e são determinantes e preditoras do comportamento humano (ROKEACH; KLIEJUNAS, 1972). Atitudes desempenham um papel importante na modelagem do comportamento do consumidor, sendo úteis na compreensão dos motivos que o leva a comprar ou não um produto, nesse ou naquele lugar (ENGEL; BLACKWELL; MINIARD, 2000). A atitude em relação a uma marca é função: (i) das crenças do consumidor a respeito da habilidade da marca (instrumentalidade percebida), para satisfazer ou bloquear o consumo e razões de uso; e (ii) da importância relativa desses motivos (importância do valor) para ele. (SHETH; TALARZYK, 1972)

A atitude existe na mente de uma pessoa e cada atitude refere-se a algo ou a um objeto, sendo formada por meio de reforço (condicionamento instrumental), de experiência direta com o objeto ou por meio da observação do outro (aprendizagem por observação), já que cada um de nós apresenta milhares de atitudes, que são ativadas pela exposição da pessoa ao objeto. (MICHENER; DELAMATER; MYERS, 2005)

\subsection{Valores Pessoais}

Os valores pessoais são entendidos como crenças intrínsecas, duradouras e, relativamente, estáveis na vida de um indivíduo. Essas crenças são representações mentais de necessidades utilizadas pelos indivíduos como base geral para a resolução de conflitos e de decisões, pois elas determinam, regulam e modificam relações entre indivíduos, organizações e sociedades (LAGES; FERNANDES, 2005). Os valores são considerados metas desejáveis que atuam como princípios orientadores na vida das pessoas (SCHWARTZ, 1992), pois expressam os objetivos que as motivam e indicam 
o caminho para atingi-los (HOFFMANN; TEERLING, 2007). Os valores pessoais representam as crenças do consumidor sobre a vida e o comportamento aceitável das pessoas (ENGEL; BLACKWELL; MINIARD, 2000). Assim, os valores são tratados como objetivos de vida desejáveis para uma pessoa ou sociedade, cujos valores instrumentais levam aos valores terminais. (ROKEACH, 1968, 1973, 1981)

Um valor é uma crença duradoura do qual um modo de conduta específico ou um estado-final de existência é pessoal ou socialmente preferível a outro modo de conduta ou estado final de existência oposto ou contrário (ROKEACH, 1968, 1973). Baseado nesse conceito, o autor desenvolveu o modelo Rokeach Value Survey (RVS), constituído por 36 itens, sendo dividido em duas partes de medidas complementares de tipos de valores pessoais. Uma parte possui 18 valores instrumentais, que representam os modos de conduta ideais, e a outra parte possui 18 valores terminais, que representam os estados de existência ideais, ou seja, aqueles valores que buscamos na vida. O modelo RVS foi utilizado por muitos pesquisadores nacionais $e$ internacionais em diversos contextos, além de ter sido traduzido para vários idiomas, como o francês, o alemão, o vietnamita, o hebraico, entre outros. (GUNTHER, 1981)

\section{Modelo Teórico}

Vários estudos (ROKEACH, 1968; VINSON; SCOTT; LAMONT, 1977; KAHLE, 1980; BALL-
-ROKEACH; LOGES, 1994; KRISTIANSEN; ZANNA, 1994; HOMER; KAHLE, 1988; SHIM; EASTLICK, 1998; SHIM; WARRINGTON; GOLDSBERRY, 1999; ROHAN, 2000; KIM et al., 2002; SHIM; MAGGS, 2005; SAWYERR; STRAUSS; YAN, 2005) tratam as atitudes como antecedentes do comportamento humano. Como o constructo lealdade é composto, também, por uma abordagem comportamental, que é baseada em aspectos de comportamento futuro de compras repetidas, quantidade e frequência de compras; e mudanças de marcas ao longo do tempo (BANDYOPADHYAY; MARTELL, 2006), além da intenção de recompra (JONES; SASSER JR., 1995; CRONIN; BRADY; HULT, 2000; SEIDERS et al., 2005), verifica-se suporte empírico para hipotetizar o constructo atitude como antecedente da lealdade, como o estudo de Marandi, Little e Sekhon (2006).

As atitudes são organizações relativamente duradouras de crenças inter-relacionadas que descrevem, avaliam e defendem ações a respeito de objetos ou situações (ROKEACH, 1968). No entanto, "[...] as atitudes são menos globais que os sistemas de valor [...]", pois as atitudes giram em torno de um objeto ou situações predispondo um indivíduo a responder de alguma forma preferencial (SAWYERR; STRAUSS; YAN, 2005, p. 499). Dessa forma, os valores pessoais têm sido usados como variável independente para entender atitudes e comportamento (SPINI, 2003). Considerando que os indivíduos e grupos diferem quanto à importância relativa que atribuem a seus valores, apresentando prioridades e hierarquias axiológicas diferentes, significando que um valor pode ser

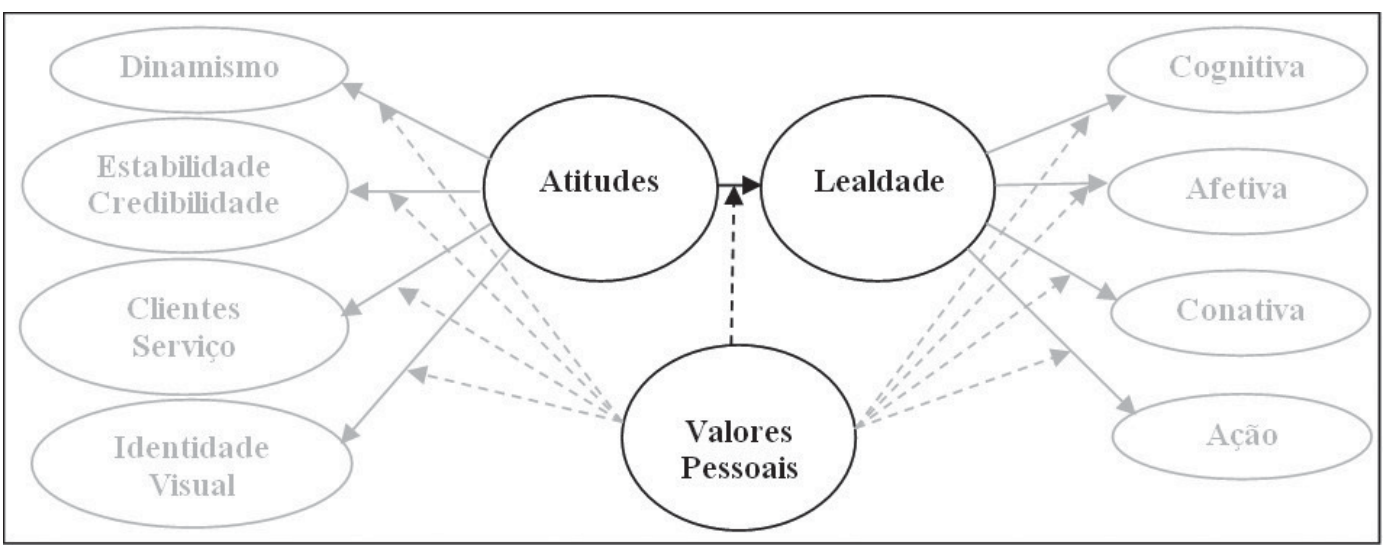

Nota: os indicadores dos constructos de segunda ordem não são apresentados por uma questão de parcimônia

Figura 1: Relação Atitudes-Fases da Lealdade moderada pelos Valores Pessoais

Fonte: Elaborado pelos autores com base na revisão de literatura 
muito importante para uma pessoa, mas de pouca importância para outra (SCHWARTZ, 1992, 2006), verifica-se que os valores pessoais podem atuar como moderadores na relação atitudes-lealdade. No efeito moderador uma variável afeta a direção e/ou a força da relação entre duas outras. (BARON; KENNY, 1986)

Assim, amparado pelos trabalhos já relacionados, propõe-se que os valores pessoais são moderadores na relação entre atitudes e fases da lealdade (Figura 1).

\section{Método}

A presente investigação da moderação dos valores pessoais na relação atitudes-fases da lealdade se baseou numa pesquisa realizada junto a clientes bancários. Para responder ao questionário, o entrevistado deveria ter um relacionamento superior a seis meses com o seu banco. $\mathrm{O}$ estudo foi realizado em duas etapas, uma exploratória, com a utilização do método levantamento de dados secundários (CHURCHILL; IACOBUCCI, 2005), para a geração dos atributos investigados na segunda etapa, de caráter quantitativo. O instrumento de coleta de dados foi dividido em quatro partes. A primeira foi composta pelos atributos de valores (18 valores terminais - RVS), numa escala tipo ordinal de 1 a 9 , sendo 1 o mais importante e 9 o de menor importância. Os valores terminais foram escolhidos por representarem os estados de existência ideais, ou seja, aqueles valores que buscamos na vida. A segunda parte foi composta por 20 atributos de atitudes, investigados numa escala de diferencial semântico, numa distância entre 1 e 5, conforme sugerido por Heerden e Puth (1995) ${ }^{1}$. A terceira parte foi composta por 16 atributos, representando as fases da lealdade, numa escala intervalar de sete pontos do tipo Likert, variando de "discordo totalmente" (1) a "concordo totalmente" (7). Os atributos das fases seguiram as escalas de Harris e Goode (2004) para as fases cognitiva e ação, de Pedersen e Nysveen (2001) para a afetiva, e de McMullan e Gilmore (2003) para a conativa. A quarta parte foi composta pelos dados demográficos dos respondentes. Os indicadores de valores e atitudes seguiram a ordem alfabética e os de lealdade tiveram suas ordens variadas. As escalas utilizadas no estudo
(Quadro 1) passaram pelo processo de tradução reversa, conforme sugerido por Gunther (1981).

\begin{tabular}{|l|l|}
\hline \multicolumn{2}{|c|}{ INDICADORES VALORES TERMinaIs } \\
\hline $\begin{array}{l}\text { Amor maduro (intimidade sexual } \\
\text { e espiritual) }\end{array}$ & $\begin{array}{l}\text { Reconhecimento social } \\
\text { (respeito e admiração) }\end{array}$ \\
\hline Autorrespeito (autoestima) & $\begin{array}{l}\text { Sabedoria (compreensão } \\
\text { amadurecida da vida) }\end{array}$ \\
\hline $\begin{array}{l}\text { Equilíbrio Interno (livre de conflito } \\
\text { interno) }\end{array}$ & $\begin{array}{l}\text { Salvação (salvar-se, vida } \\
\text { eterna) }\end{array}$ \\
\hline Felicidade (contentamento) & $\begin{array}{l}\text { Segurança da família } \\
\text { (cuidar dos seres amados) }\end{array}$ \\
\hline $\begin{array}{l}\text { Igualdade (fraternidade, } \\
\text { oportunidade igual para todos) }\end{array}$ & $\begin{array}{l}\text { Segurança nacional } \\
\text { (proteção contra ataque) }\end{array}$ \\
\hline $\begin{array}{l}\text { Liberdade (independência, } \\
\text { escolha livre) }\end{array}$ & $\begin{array}{l}\text { Sentimento de realização } \\
\text { (contribuição duradoura) }\end{array}$ \\
\hline $\begin{array}{l}\text { Mundo de beleza (beleza da } \\
\text { natureza e das artes) }\end{array}$ & $\begin{array}{l}\text { Verdadeira amizade } \\
\text { (íntimo companheirismo) }\end{array}$ \\
\hline $\begin{array}{l}\text { Mundo de paz (livre de guerra e } \\
\text { conflito) }\end{array}$ & $\begin{array}{l}\text { Vida confortável (vida } \\
\text { próspera) }\end{array}$ \\
\hline Prazer (vida agradável, ociosa) & $\begin{array}{l}\text { Vida excitante (vida } \\
\text { estimulante, ativa) }\end{array}$ \\
\hline
\end{tabular}

\begin{tabular}{|c|c|c|}
\hline \multicolumn{3}{|c|}{ Fatores e Indicadores da Atitude } \\
\hline \multirow{6}{*}{ 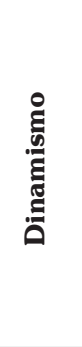 } & Sem crescimento & Crescendo rapidamente \\
\hline & Passivo & Ativo \\
\hline & Sempre progredindo & Sem muitos progressos \\
\hline & Quieto & Barulhento \\
\hline & Agressivo & Retraído \\
\hline & Rígido & Flexível \\
\hline \multirow{4}{*}{ 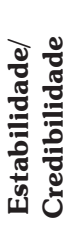 } & Confiável & Não confiável \\
\hline & Estável & Instável \\
\hline & Desonesto & Honesto \\
\hline & Clientes Positivos & Clientes Negativos \\
\hline \multirow{6}{*}{ 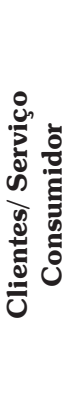 } & Amigável & Não amigável \\
\hline & $\begin{array}{l}\text { Empregados não } \\
\text { amigáveis }\end{array}$ & Empregados amigáveis \\
\hline & Bom serviço & Serviço ruim \\
\hline & Frio & Caloroso \\
\hline & $\begin{array}{l}\text { Empregados com } \\
\text { conhecimento }\end{array}$ & $\begin{array}{l}\text { Empregados sem } \\
\text { conhecimento }\end{array}$ \\
\hline & Desagradável & Agradável \\
\hline
\end{tabular}




\begin{tabular}{|c|c|c|}
\hline \multirow{4}{*}{ 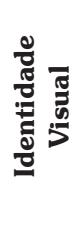 } & Não atraente & Atraente \\
\hline & Elegante & Simples \\
\hline & Invisível & Visível \\
\hline & Fora de moda & Realmente moderno \\
\hline \multicolumn{3}{|c|}{ FATORES E INDICADORES DA LEALDADE } \\
\hline \multirow{4}{*}{ 胥 } & \multicolumn{2}{|c|}{$\begin{array}{l}\text { Eu acredito que usar o Banco X é preferível a } \\
\text { outros bancos }\end{array}$} \\
\hline & \multicolumn{2}{|c|}{$\begin{array}{l}\text { Eu acredito que o Banco } \mathrm{X} \text { não oferece aquilo que } \\
\text { eu gosto }\end{array}$} \\
\hline & \multicolumn{2}{|c|}{$\begin{array}{l}\text { Eu acredito que o Banco X tem as melhores } \\
\text { ofertas neste momento }\end{array}$} \\
\hline & \multicolumn{2}{|c|}{$\begin{array}{l}\text { Eu prefiro o serviço do Banco X ao serviço de seus } \\
\text { concorrentes }\end{array}$} \\
\hline
\end{tabular}

Eu tenho uma relação emocional positiva com o Banco X

$?$ Eu me sinto ligado ao Banco X

Futuramente, eu gostaria de permanecer cliente do Banco X

O Banco X tem significado pessoal para mim

Eu experimentaria outro Banco se ele fosse $25 \%$ mais barato que o Banco $\mathrm{X}$

Eu mudaria de Banco se outro oferecesse mais status

Eu experimentaria outro banco se ele oferecesse instalações melhores que o Banco X

Eu mudaria de Banco se a equipe de outro banco fosse mais simpática

Eu sempre continuaria a escolher o Banco X antes de outros bancos

Eu sempre continuaria a favorecer as ofertas do

胥 Banco X antes de outros bancos

Eu sempre escolherei usar o Banco X preferencialmente aos bancos concorrentes

Eu sempre continuarei a escolher a qualidade do Banco X antes de outros

Quadro 1: Fatores e Indicadores dos Valores Pessoais, Atitudes e Lealdade

Fonte: Elaborado pelos autores deste artigo com base na revisão de literatura

Apenas cinco itens reversos foram usados no estudo, todos no constructo lealdade. Após a inversão dos itens e dos valores pessoais, ficando o de maior importância com o valor de 9, todo o instrumento ficou padronizado de tal modo que as notas maiores indicavam maior valor, maior atitude e maior lealdade.
A amostra pesquisada totalizou 1.001 casos respondidos, do total de 1.200 questionários distribuídos por um processo do tipo "bola de neve" (MALHOTRA, 2001). No ato do recebimento dos questionários eram checadas as respondas e eliminados aqueles que apresentassem valores ausentes (missing values), ficando a base de dados com 910 questionários válidos. Nessa base foi conduzida a análise de valores extremos (outliers), iniciando pela reposição dos casos extremos, por meio do procedimento $Z$ e intervalo interquartil, conforme sugerem Kline (1998) e Tabachinik e Fidell (2001). Dessa forma, foi possível identificar e tratar 34 outliers univariados. Em sequência identificando os outliers multivariados pela distância de Mahalanobis, conforme sugerem Hair et al. (1998), 95 respondentes foram classificados enquanto casos extremos $(p<0,001)$, considerando todas as variáveis de escala do estudo. Decidiu-se reter os casos extremos multivariados e verificar se tais respondentes distorcem os resultados das análises de modo a se evidenciar a necessidade de excluí-los das análises, garantindo uma razão justa de casos na amostra para testar os modelos. Portanto, após as etapas de purificação, a base permaneceu com 910 casos, que foram usados nas análises subsequentes.

Pela análise de normalidade observou-se, pelos testes K-S de normalidade, que as variáveis não seguem uma distribuição normal. Não se considera que tal procedimento implica na proibição da aplicação de métodos de estimação em equações estruturais que não supõem normalidade multivariada, pois o tamanho da amostra é relativamente grande, os desvios da normalidade não são expressivos e a complexidade do modelo é moderada. (TABACHINIK; FIDELL, 2001)

$\mathrm{Na}$ análise dos dados, foram utilizadas estatísticas univariadas (média, desvio-padrão) e multivariadas, com o uso da Análise Fatorial Confirmatória (AFC). Optou-se por utilizar a Análise Fatorial Confirmatória, pois o interesse é verificar a aderência de modelos e escalas previamente estudadas ao contexto do estudo. (NETEMEYER et al., 2003)

Para identificar o efeito moderador dos valores sobre a relação Atitude e Lealdade, decidiu-se por testar a diferença entre o modelo de Atitude e Lealdade para segmentos de mercado com diferentes perfis de valores. Para tornar os dados ordinais passiveis de análise por meio de técnicas de cluster tradicionais, 
aplicou-se a transformação das escalas ordinais para uma escala percentual, conforme sugere Zorn (2003). A partir deste momento aplicou-se o método de Ward com quadrado da distância Euclidiana como medida de similaridade. Observando o crescimento da medida de similaridade dos grupos, determinou-se que três seria o ponto de corte ideal para o número de clusters. Fazendo a avaliação da estabilidade da solução de clusters ao se excluir os outliers da análise, pôde-se investigar o efeito moderador dos valores sobre a relação atitude e lealdade, por meio da estratégia de multigrupos (KLINE, 1998). Os detalhes e resultados podem ser vistos nos tópicos que seguem.

\section{Resultados}

As estatísticas descritivas das variáveis mostram que os itens de lealdade tiveram média variando entre 5,27 (Eu mudaria de Banco se outro oferecesse mais status) e 3,44 (Eu experimentaria outro Banco se ele fosse $25 \%$ mais barato que o Banco X) itens do construto Lealdade Conativa. Para os itens de atitude, os valores variaram entre 4,12 (Confiável - Não confiável) e 2,99 (Agressivo - Retraído). Após a avaliação inicial dos pressupostos das técnicas procedeu-se a avaliação da fidedignidade das mensurações, por meio da Análise Fatorial Confirmatória (AFC).

A AFC seguiu duas etapas: análise do modelo de mensuração e análise do modelo estrutural, considerando a amostra completa do estudo. Na primeira delas, foi realizada a validade de construto, visando avaliar a qualidade geral da mensuração das escalas a partir dos métodos propostos por Fornell e Larcker (1981). O primeiro componente da validade de construto, a validade convergente, foi feita por meio da avaliação: (i) dos pesos padronizados entre constructos $e$ indicadores, (ii) da Confiabilidade Composta (CC) e (iii) da Variância Extraída (AVE). Estimando o modelo por meio do método de máxima verossimilhança, dado que a amostra é maior que 500 e os desvios da normalidade são moderados (TABACHINIK; FIDELL, 2001), foi possível purificar as escalas do estudo. Importa salientar que os construtos Lealdade e Atitudes foram classificados enquanto fatores de segunda ordem, fixando sua variância à unidade e mantendo a correlação entre eles livre. Considerando o limite de confiabilidade de indicadores de $40 \%$ sugerida por
Bollen (1989), foi possível reduzir o numero de indicadores de 36 a 25. Dessa forma, itens com peso padronizado inferior a 0,4 foram excluídos e as estatísticas de CC e AVE foram recalculadas. A solução reduzida obteve validade discriminante, segundo a proposta de Fornell e Larcker (1981), pois a correlação entre atitude e lealdade foi de $0,62(\mathrm{R} 2=0,38)$ e os valores da variância média extraída foram de 0,67 e 0,71 para os construtos respectivamente. Esses resultados são apresentados na Tabela 1.

Tabela 1: Medidas de Fidedignidade da Mensuração dos Constructos

\begin{tabular}{|l|c|c|c|}
\hline \multicolumn{1}{|c|}{ Constructos } & $\begin{array}{c}\text { AlPHA } \\
\text { CRonich }\end{array}$ & AVE & CC \\
\hline ATITUDE & - & 0,67 & 0,89 \\
\hline Dinamismo & 0,71 & 0,47 & 0,72 \\
\hline Estabilidade & 0,59 & 0,42 & 0,59 \\
\hline Identidade & 0,72 & 0,50 & 0,74 \\
\hline Serviço & 0,73 & 0,41 & 0,73 \\
\hline LEALDADE & - & 0,71 & 0,90 \\
\hline Lealdade Ação & 0,86 & 0,61 & 0,86 \\
\hline Lealdade Afetiva & 0,85 & 0,59 & 0,85 \\
\hline Lealdade Cognitiva & 0,73 & 0,47 & 0,73 \\
\hline Lealdade Conativa & 0,63 & 0,46 & 0,63 \\
\hline
\end{tabular}

Notas - (1) AVE - Variância Média Extraída; (2) CC - Confiabilidade Composta.

Fonte: Elaborada pelos autores deste artigo com base na análise dos dados da pesquisa

Em termos da variância média extraída (AVE) dos construtos, todos obtiveram valores superiores a 0,40, sugerido por Bollen (1989), mas considerando os valores de 0,60 para confiabilidade composta (CC) o construto Estabilidade obteve resultados pouco abaixo dos limites sugeridos, sendo, portanto, considerado válido. Considerando tais fatores em conjunto testou-se o modelo estrutural visando a identificar a validade convergente do modelo. Os resultados foram considerados satisfatórios, pois o peso de atitude para lealdade foi significativo, com valor padronizado de 0,62 ( $p<0,001)$ e os índices foram considerados válidos (GFI $=0,89 ; \chi 2 / \mathrm{df}=5,02 ;$ RMSEA $=0,07$ ), mesmo que o valor do qui-quadrado normalizado esteja um pouco acima dos limites mais liberais de 5,00 (HAIR et al., 1998). Salienta-se que os resultados testados 
com e sem outliers produziu conclusões e índices de ajuste similares, de modo, pode-se atestar que tais casos não são ofensivos para estimativas do estudo. Antes de prosseguir ao teste do efeito moderador de valores sobre a relação atitude-lealdade, apresentam-se os resultados dos clusters encontrados. A Tabela 2 apresenta os resultados das médias dos segmentos na escala de 0 a 100, transformadas para o estudo.

Observa-se que os valores agrupados nos três segmentos aproximam-se dos conceitos autopromoção, hedonismo e autotranscendência, definidos por Schwartz $(1992,2006)$ na consolidação da estrutura teórica de relações entre valores.

Tabela 2: Medidas de Comparação entre as Médias dos Segmentos

\begin{tabular}{|c|c|c|c|}
\hline \multirow[b]{2}{*}{ VALORES } & \multicolumn{3}{|c|}{ Segmentos } \\
\hline & 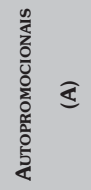 & 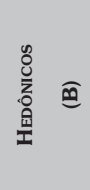 & 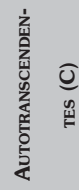 \\
\hline Sabedoria & $64^{\mathrm{bc}}$ & 42 & 42 \\
\hline Autorrespeito & $60^{\mathrm{bc}}$ & 46 & 41 \\
\hline Equilibrio Interno & $63^{b c}$ & 41 & 46 \\
\hline Amor Maduro & $58^{\mathrm{bc}}$ & $50^{c}$ & 37 \\
\hline Sentimento de Realização & $55^{c}$ & $52^{c}$ & 38 \\
\hline Vida Excitante & 51 & 50 & 47 \\
\hline Vida Confortável & $45^{c}$ & $60^{\mathrm{ac}}$ & 37 \\
\hline Felicidade & 39 & $61^{\mathrm{ac}}$ & $46^{\mathrm{a}}$ \\
\hline Reconhecimento Social & 42 & $58^{\mathrm{ac}}$ & 46 \\
\hline Prazer & 48 & $55^{\mathrm{ac}}$ & 43 \\
\hline Igualdade & $47^{\mathrm{b}}$ & 41 & $76^{\mathrm{ab}}$ \\
\hline Mundo de Paz & 38 & $49^{a}$ & $73^{\mathrm{ab}}$ \\
\hline Liberdade & 50 & 48 & $55^{\mathrm{b}}$ \\
\hline Segurança da Família & 42 & $54^{\mathrm{a}}$ & $55^{\mathrm{a}}$ \\
\hline Segurança Nacional & 48 & 49 & $55^{\mathrm{ab}}$ \\
\hline Salvação & 48 & 50 & $53^{\mathrm{a}}$ \\
\hline Verdadeira Amizade & $53^{b}$ & 46 & $53^{\mathrm{b}}$ \\
\hline Mundo de Beleza & 50 & 50 & 50 \\
\hline
\end{tabular}

Nota - as letras "a", "b" e "c" ao lado das médias indicam que o valor é diferente daquele grupo indicado. Os resultados se baseiam em testes t para amostras independentes (alfa $=5 \%$ ) e a correção de Bonferroni.

Fonte: Elaborada pelos autores deste artigo com base na análise dos dados da pesquisa
O Segmento A, que tem maiores escores em valores que representam um foco no equilíbrio interno $e$ desenvolvimento pessoal, se aproxima do conceito autopromoção, que representa os valores de poder (status social e prestígio, controle ou domínio sobre pessoas e recursos) e os valores de conquista (sucesso pessoal por meio de demonstração de competência de acordo com padrões sociais), ambos enfatizando a superioridade social, a autoestima e a busca do autointeresse. Dessa forma, os indivíduos que se encontram no Segmento A foram denominados Autopromocionais.

O Segmento B, que tem maiores escores nos aspectos de conforto, felicidade, reconhecimento social e prazer pessoal, se aproxima do conceito hedonismo, que representam o prazer $e$ a gratificação prazerosa de si mesmo. Assim, os indivíduos que se encontram no Segmento B foram denominados Hedônicos.

O Segmento $C$, que tem seus escores em valores que representam preocupação superior com aspectos globais, como igualdade e segurança, se aproxima do conceito autotranscendência, que representa os valores universalismo (compreensão, agradecimento, tolerância, proteção do bem-estar de todas as pessoas $e$ da natureza) e benevolência (preservação e fortalecimento do bem-estar daqueles do grupo "interno"), ambos lidando com a melhoria dos outros e a transcendência de interesses egoístas. Assim, os indivíduos que se encontram no Segmento $C$ foram denominados

\section{Autotranscendentes.}

Observam-se médias significativamente diferentes entre os perfis demográficos dos grupos (segundo testes qui-quadrado, Kruskal-Wallis e Mann-Whitney com alpha $=0,05$ ).

O Segmento A tem a maior proporção de mulheres (56\%), maior proporção de indivíduos casados (49\%) e é composto por pessoas mais velhas que os Segmentos B e C. Em comparação ao Segmento C, o Segmento A possui maior renda, idade e escolaridade. O Segmento B tem a maior proporção de homens (55\%), menor renda média e maior proporção de indivíduos solteiros (55\%) que o Segmento A; e a maior proporção de profissionais liberais (13\%) e estudantes (21\%) dentre os segmentos. Finalmente o Segmento $\mathrm{C}$ tem maior proporção de solteiros que o Segmento A $(52 \%)$ e menor escolaridade que o Segmento B. Deste modo, atesta-se que os segmentos apresentam diferenças entre as variáveis de classificação externas 
ao processo de cluster, demonstrando a validade do processo. (HAIR et al., 1998)

\subsection{Efeito Moderador dos Valores Pessoais}

O modelo teórico apresentado foi submetido à análise de equações estruturais, aplicando o procedimento usual de teste de modelos multigrupos (NETEMEYER et al., 2003). Nesses modelos, o valor total da estatística qui-quadrado foi assumido como a base de comparação, e os modelos adicionais foram criados por meio de restrições às matrizes do modelo de mensuração, visando avaliar a equivalência métrica das escalas entre os grupos (NETEMEYER et al., 2003). Como se trata de modelos aninhados (HAIR et al., 1998), é possível comparar o aumento da estatística qui-quadrado em relação ao modelo irrestrito (KLINE, 1998), verificando a equivalência dos grupos, conforme mostra a Tabela 3.

Tabela 3: Testes de Equivalência de Mensuração do Modelo por Segmento

\begin{tabular}{|c|c|c|c|c|c|}
\hline $\begin{array}{l}\text { Modelo } \\
\text { Testado }\end{array}$ & $\chi^{2}$ & GL. & $\begin{array}{c}\text { DIF. } \\
\chi^{2}\end{array}$ & $\begin{array}{c}\text { DIF. } \\
\text { GL }\end{array}$ & SIG. \\
\hline $\begin{array}{l}\text { Irrestrito } \\
\text { (modelo base) }\end{array}$ & 2017,12 & 798 & & & \\
\hline $\begin{array}{l}\text { Erros de } \\
\text { indicadores } \\
\text { equivalentes }\end{array}$ & 2107,93 & 848 & 90,81 & 50 & 0,00 \\
\hline A-B & 2049,52 & 823 & 32,40 & 25 & 0,15 \\
\hline A-C & 2080,60 & 823 & 63,48 & 25 & 0,00 \\
\hline B-C & 2059,65 & 823 & 42,53 & 25 & 0,02 \\
\hline $\begin{array}{l}\text { Pesos de } \\
\text { atitude } \\
\text { equivalentes }\end{array}$ & 2027,34 & 806 & 10,22 & 8 & 0,25 \\
\hline $\begin{array}{l}\text { Erros das } \\
\text { latentes } \\
\text { equivalentes }\end{array}$ & 2041,62 & 814 & 24,50 & 16 & 0,08 \\
\hline $\begin{array}{l}\text { Pesos de } \\
\text { lealdade } \\
\text { equivalentes }\end{array}$ & 2029,76 & 806 & 12,64 & 8 & 0,12 \\
\hline $\begin{array}{l}\text { Pesos } \\
\text { indicadores } \\
\text { dos construtos } \\
\text { equivalentes }\end{array}$ & 2058,78 & 832 & 41,66 & 34 & 0,17 \\
\hline
\end{tabular}

Nota -DIF. GL corresponde à diferença no valor qui-quadrado entre os modelos restritos e o modelo irrestrito. A significância testa a hipótese de que os modelos testados são equivalentes.

Fonte: Elaborada pelos autores deste artigo com base na análise dos dados da pesquisa
Na Tabela 3 observa-se que os segmentos são estatisticamente equivalentes em todos os parâmetros do modelo, exceção feita aos erros de mensuração dos indicadores dos construtos. Para essa matriz foi feita a comparação pareada dos segmentos, já que foram encontradas diferenças entre os grupos A-C e B-C: tal resultado implica que o grupo $\mathrm{C}$ apresenta erros de mensuração diferentes dos segmentos $\mathrm{A} e$ B. Observando os valores das matrizes nota-se que o segmento $\mathrm{C}$ apresenta maiores erros de mensuração em seus indicadores, o que pode ser explicado devido à menor escolaridade do grupo, o que implicaria em maior dificuldade no entendimento do instrumento de pesquisa, traduzindo maiores erros de mensuração. Assim, estimou-se um modelo restrito em que todos os parâmetros foram classificados enquanto equivalentes entre os segmentos, exceto os erros de mensuração dos indicadores, estimados livremente para o segmento C.

Em sequência este modelo foi usado para comparar o peso estrutural de atitude para lealdade. O modelo base com $\chi^{2}=2.204,62$ e 890 graus de liberdade não teve diferenças significativas do modelo em que os pesos de atitude para lealdade foram estimados de maneira livre entre os grupos $\left(\chi^{2}=2.205,09\right.$ e 892; sig. $=0,79)$ de modo que se pode atestar que não foram detectadas diferenças significativas entre o peso de atitude e fases da lealdade entre os segmentos.

\subsection{Comparação de Médias de Atitude e Fases da Lealdade entre os Segmentos}

Percebeu-se pelas análises anteriores que as $\underline{\underline{r}-}$ lações entre as variáveis do modelo não foram significativamente diferentes entre os grupos. Como análises adicionais, verificamos em que medida as respostas dos pesquisados para atitudes e lealdade diferem quando se consideram os segmentos com valores distintos. Em outras palavras, podemos perguntar 'as atitudes dos consumidores são diferentes entre os que possuem valores diferentes?'. Da mesma forma, 'a lealdade, mais especificamente as diferentes fases da lealdade, são diferentes entre os grupos com valores distintos?'

$\mathrm{Na}$ Tabela 4 são apresentados os resultados para as dimensões de atitude. Nas quatro dimensões de atitude investigadas, observou-se que não foram encontradas diferenças significativas. As médias foram muito similares dentro dos grupos. Isso significa que 
os clusters têm uma atitude similar em relação ao seu banco de maior relacionamento.

Tabela 4: Comparação de médias de Atitudes dentre os clusters de valores

\begin{tabular}{|c|c|c|c|c|c|}
\hline Dimensóes & $\begin{array}{c}\text { Segmen- } \\
\text { TOS }\end{array}$ & $\begin{array}{c}\text { Amos- } \\
\text { TRA }\end{array}$ & $\begin{array}{l}\text { MÉ- } \\
\text { DIA }\end{array}$ & $\begin{array}{c}\text { Teste } \\
\text { F }\end{array}$ & SIG \\
\hline \multirow{3}{*}{ Dinamismo } & A & 251 & 3,64 & \multirow{3}{*}{0,789} & \multirow{3}{*}{0,454} \\
\hline & B & 388 & 3,61 & & \\
\hline & $\mathrm{C}$ & 271 & 3,68 & & \\
\hline \multirow{3}{*}{ Estabilidade } & A & 251 & 3,64 & \multirow{3}{*}{0,113} & \multirow{3}{*}{0,893} \\
\hline & B & 388 & 4,12 & & \\
\hline & C & 271 & 4,12 & & \\
\hline \multirow{3}{*}{ Serviço } & A & 251 & 4,10 & \multirow{3}{*}{0,302} & \multirow{3}{*}{0,739} \\
\hline & B & 388 & 4,11 & & \\
\hline & C & 271 & 3,50 & & \\
\hline \multirow{3}{*}{ Identidade } & A & 251 & 3,49 & \multirow{3}{*}{0,005} & \multirow{3}{*}{0,995} \\
\hline & B & 388 & 3,53 & & \\
\hline & C & 271 & 3,50 & & \\
\hline
\end{tabular}

Fonte: Elaborada pelos autores deste artigo com base na análise dos dados da pesquisa

Por outro lado, os resultados foram diferentes quanto à avaliação da lealdade pelos participantes. Esses resultados são apresentados na Tabela 5. Nota-se que a lealdade cognitiva apresentou média significa- tivamente maior no grupo autopromocionais (média $=4,70$ ) quando comparados ao grupo autotranscendentes (média $=4,43$, sig. $=0,056$ ). Essa diferença se repetiu na lealdade afetiva, com médias de 4,72 e 4,38 entre os grupos (sig. $=0,048$ ). Na lealdade de ação, houve diferença entre os segmentos autopromocionais e hedônicos, embora no nível de 10\% de significância, sendo que a média de lealdade foi relativamente maior para o grupo dos autopromocionais.

\section{Discussão e Considerações}

A lealdade do consumidor é um importante constructo do marketing, pois possibilita mensurar a relação cliente-empresa, permitindo estabelecer um relacionamento que gere aumento dos lucros e retenção de clientes (KUMAR; SHAH, 2004). O fenômeno da lealdade do consumidor é investigado desde o começo do século passado (HOMBURG; GIERING, 2001) e, segundo Oliver (1997, 1999), essa lealdade é constituída por fases (afetiva, cognitiva, conativa e ação), que não emergem de maneira simultânea, mas consecutivamente no decorrer do tempo. Esse modelo proposto por Oliver só recentemente tem sido sujeito a testes empíricos extensivos. (EVANSCHITZKY; WUNDERLICH, 2006)

Tabela 5: Comparação de médias de Lealdade dentre os clusters de valores

\begin{tabular}{|c|c|c|c|c|c|c|}
\hline Dimensões & Segmentos & Амоstra & MÉDIA & Teste $\mathbf{F}$ & Sig & DifERENÇA ENTRE SEGMENTOS ${ }^{A}$ \\
\hline \multirow{3}{*}{ Lealdade Cognitiva } & A & 251 & 4,70 & \multirow{3}{*}{3,166} & \multirow{3}{*}{0,043} & \multirow{3}{*}{ A vs. $C$ (sig. $=0,056)$} \\
\hline & B & 388 & 4,63 & & & \\
\hline & $\mathrm{C}$ & 271 & 4,43 & & & \\
\hline \multirow{3}{*}{ Lealdade Afetiva } & A & 251 & 4,72 & \multirow{3}{*}{3,272} & \multirow{3}{*}{0,038} & \multirow{3}{*}{ A vs. $C$ (sig. $=0,048)$} \\
\hline & B & 388 & 4,47 & & & \\
\hline & $\mathrm{C}$ & 271 & 4,38 & & & \\
\hline \multirow{3}{*}{ Lealdade Conativa } & A & 251 & 4,80 & \multirow{3}{*}{2,241} & \multirow{3}{*}{0,107} & \multirow{3}{*}{ ns } \\
\hline & B & 388 & 4,67 & & & \\
\hline & $\mathrm{C}$ & 271 & 4,53 & & & \\
\hline \multirow{3}{*}{ Lealdade de Ação } & A & 251 & 4,57 & \multirow{3}{*}{2,812} & \multirow{3}{*}{0,061} & \multirow{3}{*}{ A vs. $B($ sig. $=0,077)$} \\
\hline & B & 388 & 4,29 & & & \\
\hline & $\mathrm{C}$ & 271 & 4,33 & & & \\
\hline
\end{tabular}

Nota - (a) Diferenças significativas entre os pares, segundo teste de Scheffe; (ns) não houve diferença entre os grupos

Fonte: Elaborada pelos autores deste artigo com base na análise dos dados da pesquisa 
Encontram-se, na literatura, vários estudos sobre fatores que afetam a lealdade, tanto mediadores, como qualidade (TSIOTSOU, 2006), quanto moderadores, como satisfação (HOMBURG; GIERING, 2001). No entanto, mesmo com uma vasta literatura sobre o tema, nota-se uma lacuna quanto à relação dos valores pessoais, atitudes e fases da lealdade, proposta por Oliver (1997, 1999). Com base nesta constatação, teve-se por objetivo nessa pesquisa testar o papel moderador dos valores pessoais na relação entre atitudes e as fases da lealdade, junto a clientes bancários.

Os resultados principais mostraram o constructo atitudes como antecedente das fases da lealdade. Já o papel moderador dos diferentes segmentos baseados nos valores pessoais (Autopromocionais, Hedônicos e Autotranscendentes) não se mostrou significativo no modelo estrutural. No entanto, na comparação de médias de lealdade dentre os clusters, pode-se destacar que (i) os autopromocionais se diferenciam dos autotranscendentes, nas fases cognitiva e afetiva, e (ii) os autopromocionais se diferenciam dos hedônicos na fase ação.

No Modelo Estrutural, as atitudes se confirmaram como preditoras das fases da lealdade, confirmando as atitudes como antecedentes do comportamento humano. No entanto, a moderação pelos valores foi estatisticamente equivalente em termos de todos os parâmetros do modelo, entre os três grupos, exceção feita aos erros de mensuração dos indicadores dos construtos. Ou seja, a moderação realizada de forma simultânea e comparativa não apresenta diferenças entre os grupos, constatando que os valores pessoais não interferem na força da relação atitudes-fases da lealdade. Quanto à exceção, erros de mensuração ocorridos com o segmento autotranscendentes, podem estar relacionados às características do grupo, que não é preocupado com questões específicas, tais como conhecimento, afeto ou intenção de compras, mas sim com questões gerais e mais globais.

Na comparação das médias de lealdade dentre os clusters, observa-se que há diferenças entre os grupos autopromocionais e autotranscendentes, nas fases cognitiva e afetiva, e entre os grupos autopromocionais e hedônicos, na fase ação. O segmento dos autopromocionais tem a maior proporção de mulheres, de indivíduos casados e é composto por pessoas mais velhas do que os hedônicos e autotranscendentes. $\mathrm{O}$ segmento dos autotranscendentes é composto por mais homens, mais jovens, mais solteiros, de menor renda e de menor escolaridade se comparado ao segmento dos autopromocionais. Assim, os indivíduos do grupamento autopromocionais, talvez, por serem distintos e por possuírem características focadas em si, têm fundamentos internos e externos (HOMER; KAHLE, 1988) que os influenciam nas dimensões cognitiva, afetiva e ação da lealdade. Logo, (i) os indivíduos classificados como autopromocionais têm uma estrutura de valores mais orientada interna e externamente para o conhecimento de uma marca, o afeto a essa marca e a ação de comprá-la, ou seja, tendem mais à lealdade, (ii) os indivíduos que estão orientados para questões mais globais, como os autotranscendentes, tendem menos à lealdade cognitiva $e$ afetiva, se comparados aos autopromocionais, e (iii) os indivíduos que têm foco em questões prazerosas, como os hedônicos, tendem menos à lealdade ação, se comparados aos autopromocionais.

Os resultados dessa pesquisa vão ao encontro de dois estudos, o de Giacomino e Eaton (2003), quanto à caracterização dos autotranscendentes, respondentes mais jovens que mostraram uma maior orientação em relação a servir aos outros e em relação aos meios morais, e os estudos de Sawyerr, Straus e Yan (2005), que ao estudarem a estrutura de valores e a atitude de aceitação das semelhanças e das diferenças existentes entre as pessoas, constataram que os indivíduos com valores de autopromoção se opõem aos indivíduos de valores de autotranscendência.

Em resumo, algumas contribuições teóricas relevantes emergem com os resultados desse trabalho. A primeira é identificar que indivíduos que compartilham dos mesmos valores, segmentos identificados, agem de forma semelhante em relação às três fases da lealdade, propostas por Oliver (cognitiva, afetiva e ação). A segunda é constatar que os mais jovens tendem a ser preocupar com questões mais globais, podendo ser caracterizados como socialmente conscientes. Por fim, que não há diferenças entre segmentos quando se comparam relações entre os constructos do modelo (atitudes $\rightarrow$ lealdade), mas há diferenças, entre os segmentos, nas avaliações feitas pelos pesquisados sobre as fases da lealdade, conforme visto na comparação de médias por ANOVA. 
Quanto ao contexto financeiro, constata-se que quanto maior a atitude, representada na pesquisa por fatores de imagem, maior a lealdade do consumidor, conforme confirmado no modelo estrutural. Dessa forma, cabe aos administradores fortalecerem a imagem da instituição, destacando fatores como dinamismo, identidade e serviços, a fim de estabelecer relacionamentos duradouros.

Outra implicação gerencial a ser explorada é o fato de o segmento autopromocionais tender mais à lealdade cognitiva e afetiva do que o segmento autotranscendentes, e mais à lealdade ação do que o segmento hedônicos. Assim, as instituições financeiras devem estabelecer estratégias para identificar, junto às suas bases de clientes, as características que marcam o primeiro segmento e, então, explorar as fases da lealdade. Lembrando que a cognição pode ter origem a partir de conhecimento prévio ou mesmo adquirida pela experiência pessoal com o produto/serviço, a afetiva está relacionada a uma atitude positiva em relação à marca que se origina a partir da satisfação cumulativa com repetidas situações de compra e uso, e a ação, que se baseia na pré-disposição de agir e na superação de obstáculos de compra (OLIVER, 1997, 1999). Dessa forma, as empresas poderão manter seus consumidores e estabelecer relacionamentos de longo prazo, especialmente, com aqueles "bem resolvidos" e determinados.

Em síntese, os resultados desta pesquisa permitem afirmar que: (i) o constructo atitudes é um antecedente significativo das fases da lealdade, (ii) não há efeito moderador dos segmentos de valores pessoais na relação entre atitudes e fases da lealdade, e (iii) quando comparadas as médias da lealdade, verifica-se que há diferenças nas fases cognitiva, afetiva e ação entre os segmentos baseados nos valores pessoais.

Dentre as limitações, nota-se que a mensuração dos constructos valores pessoais pode ser diferente em pesquisas futuras. Por exemplo, nessa pesquisa foram utilizados os valores terminais da escala Rokeach Value Survey (RVS), em futuros trabalhos pode-se utilizar a escala RVS completa (valores instrumentais e valores terminais) ou outras escalas de valores validadas, como Values and Life Style (VALS), List of Values (LOV), entre outras, e, dessa forma, comparar o papel moderador dos valores na relação atitude-fases da lealdade. Outro fator, ainda referente aos valores pessoais, é o método utilizado nessa pesquisa, que foi o ranking, sugere-se para futuras pesquisas utilizar o critério de rating e avaliar se os resultados são convergentes aos encontrados nesse estudo. Sugere-se, ainda, em novos trabalhos, testar modelos concorrentes, com os constructos valores pessoais, atitudes e fases da lealdade em diferentes papéis, como o efeito direto dos valores pessoais nas fases da lealdade e/ou os efeitos mediadores e moderadores das atitudes na relação valores pessoais-fases da lealdade. Tais investigações contribuirão para ampliar o conhecimento sobre a lealdade do consumidor.

\section{REFERÊNCIAS}

BAGOZZI, Richard. Marketing as an organized behavioral system of exchange. Journal of Marketing, Chicago, USA, v. 38, p. 77-81, 1974.

BALL-ROKEACH, Sandra J.; LOGES, William E. Choosing equality: the correspondence between attitudes about race and the value of equality. Journal of Social Issues, New Jersey, USA, v. 50, n. 4, p. 9-18, 1994.

BANDYOPADHYAY, S.; MARTELL, M. Does attitudinal influence behavioral loyalty? A theoretical and empirical study. Journal of Retailing and Consumer Services, Philadelphia, USA, v. 14, n. 1, p. 35-44, 2006.

BARON, R. M.; KENNY, D. A. The moderator-mediator variable distinction in social psychological research: conceptual, strategic, and statistical considerations.

\section{Journal of Personality and Social Psychology,} Washington, USA, v. 51, n. 6, p. 1.173-1.182, 1986.

BEATTY, Sharon E. et al. Alternative measurement approaches to consumer values: the list of values and Rokeach Value Survey. Psychology and Marketing, New Jersey, USA, v. 2, p. 181-200, 1985.

BENNETT, R.; RUNDLE-THIELE, S. A Comparison of attitudinal loyalty measurement approaches. Journal Brand Management, Hampshire, UK, v. 9, n. 3, p. 193209, 2002.

\section{BOLLEN, K. A. Structural equations with latent}

variables. New York: John Willey \& Sons, 1989. 
CARUANA, A. The impact of switching costs on customer loyalty: a study among corporate customers of mobile tlephony. Journal of Targeting, Measurement and Analysis fo Marketing, Hampshire, UK, v. 12, n. 3, p. 256-268, 2004

CHAUDHURI, A.; HOLBROOK, M. The chain of effects from brand trust and brand affect to brand performance: the role of brand loyalty. Journal of Marketing, Chicago, USA, v. 65, n. 2, p. 81-93, 2001.

CHURCHILL JR., Gilbert A.; IACOBUCCI, Dawn.

Marketing research: methodological foundations. Ohio: Thomson South-Western, 2005.

CRONIN, J.; BRADY, M. K.; HULT, G. T. Assessing the effects of quality, value, and customer satisfaction on behavioral intention in service environments. Journal of Retailing, New York, USA, v. 76, n. 2, p. 193-218, 2000.

DWYER, R.; SCHURR, P.; OH, S. Developing buyer-seller relationships. Journal of Marketing, Chicago, USA, v. 51, n. 2, p. 11-27, 1987.

ENGEL, James F.; BLACKWELL Roger D.; MINIARD Paul W. Comportamento do consumidor. 8. ed. Rio de Janeiro: Livros Técnicos Científicos S.A., 2000. 641p.

EVANSCHITZKY, Heiner; WUNDERLICH, M. An examination of moderator effects in the four-stage loyalty model. Journal of Service Research, California, USA, v. 8, n. 4 , p. $330-345,2006$.

FORNELL, C.; LARCKER, D. F. Evaluating structural equation models with unobservablevariables and measurement error. Journal of Marketing Research, Chicago, USA, v. 18, p. 39-50, 1981.

FORNELL, C. et al. The american customer satisfaction index: nature, purpose, and findings. Journal of Marketing, Chicago, USA, v. 60, n. 4, p. 7-18, 1996.

GARBARINO, Ellen; JOHNSON, Mark. The different roles of satisfaction, trust and commitment for relational and transactional consumers. Journal of Marketing, v. 63, n. 2, p. 70-87, Apr. 1999.

GIACOMINO, Don E.; EATON, Tim V. Personal values of accounting alumi: an empirical examination of differences by gender and age. Journal of Managerial Issues, Chicago, USA, v. 15. n. 3, p. 369-380, 2003.
GRUBE, Joel W.; MAYTON II, Daniel M.; BALL$\mathrm{ROKEACH}$, Sandra J. Inducing change in values, attitudes, and behaviors: belief system theory and the method of value sef-confrontation. Journal of Social Issues, New Jersey, USA, v. 50, n. 4, p. 153-173, 1994.

GUNTHER, Hartmut Ein. Uma tentativa de traduzir e adaptar a escala de valores de rokeach para uso no Brasil. Arquivos Brasileiros de Psicologia, Rio de Janeiro, Brasil, v. 33, n. 3, p. 58-72, 1981.

HAIR, J. et al. Multivariate data analysis. 5. ed. New Jersey: Prentice Hall, 1998.

HARRIS, L. C.; GOODE, M. M. H. The four levels of loyalty and the pivotal role of trust: a study of online service dynamics. Journal of Retailing, Philadelphia, USA, v. 80, n. 2, p. 139-158, 2004.

HEERDEN, Cornelius H. Van; PUTH, Gustav. Factors that determine the corporate image of South African banking institutions: an exploratory investigation. International Journal of Bank Marketing, Bingley, United Kingdom, v. 13, n. 3, p. 1-9, 1995 .

HOFFMANN, Arvid O. I.; TEERLING, Marije L. The impact of social networks on consumer's value attitudes systems and store loyalty. [2007].

Disponível em: <http://www.ub.rug.nl/eldoc/som/ b/04B17/04B17.pdf > . Acesso em: 5 maio 2007.

HOMBURG, C.; GIERING, A. Personal characteristic as moderators of the relationship between customer satisfaction and loyalty: an empirical analysis.

Psychology \& Marketing, New Jersey, USA, v. 18, n. 1, p. 43-56, 2001.

HOMER, Pamela M.; KAHLE, Lynn R. A Structural Equation Test of the Value-Attitude-Behavior Hierarchy. Journal of Personality and Social Psychology, Washington, USA, v. 54, n. 4, p. 638-646, 1988.

JACOBY, J.; KYNER, D. B. Brand loyalty vs. repeat purchasing behavior. Journal of Marketing Research, Chicago, USA, v. 10, n. 1, p. 1-9, 1973.

JACOBY, J.; CHESNUT, R. Brand Loyalty: measurement and management. New York: Wiley, 1978. 
JONES, T. O.; SASSER JR., W. E. Why satisfied customers defect. Harvard Business Review, Massachusetts, USA, v. 63, n. 6, p. 88-99, 1995.

JONES T. O.; TAYLOR, S. F. The conceptual domain of service loyalty: how many dimensions? Journal of Services Marketing, California, USA, v. 21, n. 1, p. 36$51,2007$.

KAHLE, Lynn R. Stimulus condition self-selection by males in the interations of locus of control and skillchance situations. Journal of Personality and Social Psychology, Washington, USA, v. 38, p. 50-56, 1980.

KIM, Jai-Ok et al. Cros-cultural consumer values, needs and purchase behavior. Journal of Consumer Marketing, New Jersey, USA, v. 19, n. 6, p. 481-502, 2002.

KLINE, R. B. Principles and practice of structural equation modeling. New York: The Guilford Press, 1998.

KRISTIANSEN, Connie M.; ZANNA, Mark P. The Rhetotical Use of Values to Justify Social and Intergroup Attitudes. Journal of Social Issues, New Jersey, USA, v. 50, n. 4 , p. $47-65,1994$.

KUMAR V.; SHAH, D. Building and sustaining profitable customer loyalty for the 21st century. Journal of Retailing, Philadelphia, USA, v. 80, n. 4, p. 317-330, 2004.

LAGES, Luis Filipe; FERNANDES, Joana Cosme. The SERPVAL scale: a multi-item instrument for measuring service personal values. Journal of Business Research, Philadelphia, USA, v. 58, p. 1.562-1.572, 2005.

LESSIG, V. P.; COPLEY, Thomas P. Consumer beliefs, atitudes and brand preferences. Academy of Marketing Science, California, USA, v. 2, n. 2, p. 357-366, 1974.

LU, Yan-Li et al. Fuzzy synthetic evaluation on customer loyalty based on analytic hierarchy process. Proceedings of The Fourth International Conference on Machine Learning and Cybernetics, Guangzhou, p. 18-21, 2005.

MALHOTRA, N. Pesquisa de marketing: uma orientação aplicada. Porto Alegre: Bookman, 2001. 718p.
MARANDI, Ebi; LITTLE, Ed; SEKHON, Yasmin. The impact of personal values on perception of service provider empathy and customer loyalty. The Business Review, Cambridge, Hampshire, UK, v. 5, n. 2, p. 339343, 2006.

MCMULLAN, R.; GILMORE, A. The conceptual development of customer loyalty measurement: a proposed scale. Journal of Targeting, Measurement and Analysis for Marketing, Hampshire, UK, v. 11, n. 3, p. 230-243, 2003.

MICHENER, H. Andrew; DELAMATER, John D.; MYERS Daniel J. Psicologia social. São Paulo: pioneira, 2005. (761p.)

MORGAN, Robert; HUNT, Shelby. The commitment-trust theory of relationship marketing. Journal of Marketing, Chicago, USA, v. 58, n. 3 , p. 20-38, jul. 1994.

NETEMEYER, R. G.; BEARDEN, W. O.; SHARMA, S. Scaling procedures: issues and applications. Thousand Oaks, California, EUA, SAGE, 2003.

OLIVER, R. L. Satisfaction: a behavioral perspective on the consumer. New York: Irwin McGraw-Hill, 1997.

OLIVER, R. L. Whence customer loyalty? Journal of Marketing, Chicago, USA, v. 63, special issue, p. 33-44, 1999.

PEDERSEN, Per P.; NYSVEEN, Herbjorn. Shopbot banking: an exploratory study of customer loyalty effects. The International Journal of Bank Marketing, Bingley United Kingdom, v. 19, n. 4/5, p. 146-155, 2001.

ROHAN, Meg J. A Rose by Any Name? The values Construct. Personality and Social Psychology

Review, New Jersey, USA, v. 40, n. 3, p. 255-277, 2000.

ROKEACH, Milton. A Theory of organization and change within value-attitude systems. Journal of Social, New Jersey, USA, v. 24, n. 1, p. 13-33, 1968.

Crenças, atitudes e valores. Rio de Janeiro:

Ed. Interciência, 1981. (178p.)

The Nature of human values. New York: Free Press, 1973. 
ROKEACH, Milton; KLIEJUNAS, Peter. Behavior as a function of attitude-toward-object and attitudetoward-situation. Journal of Personality and Social Psychology, New Jersey, USA, v. 22, n. 2, p. 194-201, 1972.

RUNDLE-THIELE, S.; MACKAY, M. M. Assessing the performance of brand loyalty measures. Journal of Services Marketing, Bingley United Kingdom, v. 15, n. 6/7, p. 529-546, 2001.

SAWYERR, Olukemi O.; STRAUSS, Judy; YAN, Jun. Indivudal value structure and diversity attitudes - The moderating effects of age, gender, race, and religiosity. Journal of Managerial Psychology, Bingley United Kingdom, v. 20, n. 6, p. 498-521, 2005.

SCHWARTZ, Shalom H. Universals in the content ans structure in the values: theoretical advances and empirical tests in 20 countries. Advances in Experimental Social Psychology, San Diego, Califórnia, v. 25, p. 1-65, 1992.

Basic human values: an overview - basic human values: theory, methods, and applications. Working Paper. [2006]. Disponível em: <www.fmag.unict. it/Allegati/convegno\%207-8-10-05/Schwartzpaper.pdf > . Acesso em: 14 nov. 2006

SEIDERS, K. et al. Do satisfied customers buy more? Examining moderating influences in a retailing context. Journal of Marketing, Chicago, USA, v. 69, n. 4, p. 26-43, 2005.

SHETH, J. N.; TALARZYK, Wayne. Perceived Instrumentality and value importance as determinants of attitudes. Journal of Marketing Research, Chicago, USA, v. 9, p. 6-9, 1972.

SHETH, J. N.; MITTAL, Banwari; NEWMAN, Brucce

I. Comportamento do cliente: indo além do comportamento do consumidor. São Paulo: Atlas, 2001. (795p.)

SHIM, S.; EASTLICK, M. A. The hierarchical influence of personal values on mall shopping attitude and behavior. Journal of Retailing, Philadelphia, USA, v. 74, n. 1, p. 139-160, 1998.
SHIM, S.; WARRINGTON, P.; GOLDSBERRY, E. A personal value-based model of college students' attitudes and expected choice behavior regarding retailing careers. The Family and Consumer Science Research Journal, New Jersey, USA, v. 28, p. 28-51, 1999.

SHIM, S.; MAGGS, Jennifer. A Cognitive and behavioral hierarchical decision-making model of college students' alcohol consumption. Psychology \& Marketing, New Jersey, USA, v. 22, n. 8, p. 649-668, 2005.

SPINI, Dario. Measurement equivalence of 10 value types from the Schwartz Value Survey across 21 countries. Journal of Cross-Cultural Psychology, California, USA, v. 34, n. 1, p. 3-23, January, 2003.

TABACHINIK, B. G.; FIDELL, L. S. Using Multivariate Statistics. 3. ed. New York: Harper Collins, 2001.

TSIOTSOU, R. The role of perceived product quality and overall satisfaction on purchase intentions. International Journal of Consumer Studies, New Jersey, USA, v. 30, n. 2, p. 207-217, 2006.

VINSON, Donald E.; SCOTT, J. E.; LAMONT, Lawrence $\mathrm{M}$. The role of personal value in marketing and consumer behavior. Journal of Marketing, Chicago, USA, v. 41, n. 2, p. 44-50, 1977.

YIM, C. K.; KANNAN, P. K. Consumer behavioral loyalty: a segmentation model and analysis. Journal of Business Research, Philadelphia, USA, v. 44, n. 2, p. 75-92, 1999.

\section{ZORN, Christopher. Agglomerative Clustering of} Rankings Data, with an Application to Prison Rodeo Events. Working paper, 2003. Disponível em: $<$ http://www.polmeth.wustl.edu/media/Paper/zorn03. pdf>. Acesso em: $10 \mathrm{dez} .2007$. 